\title{
Accuracy of Measurement for Counting and Intensity-Correlation Experiments*
}

\author{
Marvin L. Goldberger \\ Palmer Physical Laboratory, Princeton University, Princeton, New Jersey
}

AND

KENNETh M. Watson

Department of Physics and Lawrence Radiation Laboratory, University of California, Berkeley, California

(Received 15 June 1965)

\begin{abstract}
A quantum-mechanical analysis is made of the experimental accuracy to be expected for particle-counting and intensity-correlation experiments. The mean-square fluctuation for an ensemble, consisting of a large number of experiments each conducted over a time interval $T$, is calculated.
\end{abstract}

\section{INTRODUCTION}

A CRUCIAL element in the design of experiments is a careful estimate of the fluctuations to be expected in observed counting rates. These fluctuations, which determine a limitation on the accuracy of particle flux measurements, are conventionally and ordinarily quite correctly discussed in terms of purely classical concepts involving random arrival times. Recent interest in coherent and partially coherent beams and in rather elaborate correlation experiments suggests that a quantum theory of fluctuations may be useful.

The present paper is a sequel to an earlier one ${ }^{1}$ in which the theoretical basis for observing fluctuations and particle correlations was analyzed. We are concerned here with the accuracy of such measurements, namely, the counting times required to measure fluctuations and correlations to within specified limits.

We begin by considering a conventional experiment with a single detector which counts, say, $N$ particles during the course of an experiment. One ordinarily says that this observation is subject to fluctuations of order $N^{1 / 2}$. We study the quantum corrections to this estimate in Sec. III.

Our principal concern in this paper is with a study of the accuracy of experiments designed to observe fluctuations and correlations in particle beams. We have in mind such techniques as that of Hanbury-Brown and Twiss ${ }^{2}$ to study photon correlations, that of Goldberger, Lewis, and Watson ${ }^{3}$ to measure the phase of scattering amplitudes, those to measure spectral line shapes, ${ }^{4-6}$ etc.

In order to make this paper reasonably self contained, we summarize the relevant results of I.

* Research supported in part by the U. S. Air Force Office of Scientific Research, Grant No. AF-AFOSR-130-65 and in part by the U. S. Atomic Energy Commission.

${ }^{1}$ M. L. Goldberger and K. M. Watson, Phys. Rev. 137, B1410 (1965). This paper will henceforth be referred to as I.

2 R. Hanbury-Brown and R. Q. Twiss, Proc. Roy. Soc. (London) 243A, 291 (1957) and earlier papers.

${ }^{3}$ M. L. Goldberger, W. H. Lewis, and K. M. Watson, Phys. Rev. 132, 2764 (1963).

4 A. T. Forrester, J. Opt. Soc. Am. 41, 253 (1961).

${ }^{5} \mathrm{~L}$. Mandel, in Symposium on Electromagnetic Theory and Antennas (Pergamon Press, Ltd., London, 1963).

${ }^{6}$ L. Mandel, E. C. G. Sudarshan, and E. Wolf, Proc. Phys. Soc. 84, 435 (1964).

\section{NOTATION AND GENERAL DESCRIPTION}

We shall follow the notation introduced in I. During a time interval $T$, long compared with transients, particles in a beam are counted. These may be radiated from a source (like a hot gas or radioactive sample), a particle accelerator, or particles scattered by a target. In a given experiment there will be $n$ such particles, having individual wave functions $\Phi_{i}\left(\mathbf{x}_{i}, t\right)(i=1,2, \cdots n)$. Here $\mathbf{x}_{i}$ is the coordinate of the $i$ th particle and $t$ is the time. The wave function describing the beam is then, at time $t=0$,

$$
\Psi(0)=\S \prod_{i=1}^{n} \Phi_{i}\left(\mathbf{x}_{i}, 0\right)
$$

where $S$ is the appropriate symmetrization operator for Bose-Einstein or Fermi-Dirac statistics. We continue to suppose, as discussed in I, that the beam is incoherent in the sense that the $\Phi_{i}$ have random phases. ${ }^{7}$

The flux of beam particles, averaged over an ensemble of many experiments, is assumed to be constant throughout the interval $T$. At a distance $\mathbf{y}$ from some conveniently chosen reference point $O$ in the source (or target, if a scattering experiment is being considered) the flux is (see Fig. 1)

$$
F(\mathbf{y})=R_{B} / 4 \pi y^{2}
$$

where $R_{B}$ is the equivalent isotropic source intensity (expressed in particles emitted per second) in the direction of $y$. The flux of particles having an energy $\hbar \omega$ in the interval $\hbar d \omega$ is written as

$$
d F=F(\mathbf{y}) g(\omega) d \omega
$$

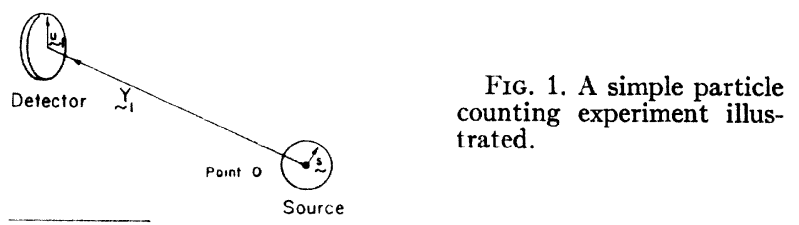

${ }^{7}$ A detailed analysis of this and of the beam wave function $\Psi(0)$ has been made by S. K. Ma (to be published). 
where the spectral function $g(\omega)$ is normalized to unity

$$
\int g(\omega) d \omega=1 .
$$

The "beam width" $\Delta \omega_{B}$ is defined as

$$
\Delta \omega_{B}=\left[\int d \omega[g(\omega)]^{2}\right]^{-1}
$$

The mean speed and momentum are written as

$$
\begin{aligned}
V & =\text { mean speed of beam particles, } \\
p & =\text { mean momentum of beam particles. }
\end{aligned}
$$

We shall assume that the beam width $\Delta \omega_{B}$ is narrow in the sense that we can neglect the dispersion in particle velocities while they are crossing the counter, or counters. By the same token wave-packet spreading may be neglected during the detection process. [The restriction to a narrow beam spectrum may be removed from our analysis with only trivial modification of our expressions as long as the detection process limits the frequency interval of coherent interference effects. An illustration of this point is provided by Eqs. (5.7) and (5.8) of I.]

Let us suppose that the beam flux is measured by a detector "1," as illustrated in Fig. 1. For simplicity, we assume that this has a uniform thickness $w_{1}$ and surface area $\Sigma_{1}$ normal to the beam direction. A convenient point in the detector will be labeled as $\mathbf{Y}_{1}$. A vector from the chosen reference point $O$ in the source to an arbitrary source point is written as s. Similarly, a vector from the point $\mathbf{Y}_{1}$ to a point in the detector is $\mathbf{u}_{1}$. We define

$$
\mathbf{y}_{1} \equiv \mathbf{Y}_{1}+\mathbf{u}_{1} \text {. }
$$

It will be convenient, but not essential to the argument, to assume in this paper that the source and detectors are small in the following sense: We shall suppose that the magnitude of the vector $D=\mathbf{u}_{1}+\mathbf{Y}_{1}-\mathbf{s}$ may be written as

$$
D \cong Y_{1}
$$

except when $D$ appears in an oscillating exponential. In the latter case, we write ${ }^{8}$

$$
\begin{aligned}
D \cong Y_{1}+\hat{Y}_{1} \cdot\left(\mathbf{u}_{1}-\mathbf{s}\right) & +\left(2 Y_{1}\right)^{-1} \\
& \times\left\{\left(\mathbf{u}_{1}-\mathbf{s}\right)^{2}-\left[\hat{Y}_{1} \cdot\left(\mathbf{u}_{1}-\mathbf{s}\right)\right]^{2}\right\} .
\end{aligned}
$$

We shall assume that the mean beam flux is uniform over the face of the detector ${ }^{9}$ so that

$$
F\left(\mathbf{y}_{1}\right) \cong F\left(\mathbf{Y}_{1}\right) \text {. }
$$

\footnotetext{
${ }^{8}$ The approximations (2.9) provide convenient algebraic simplifications for many of our expressions. The reader is cautioned, however, to verify these approximations for specific applications before using them. If Eqs. (2.9) are not valid, the general expressions may be easily written down, but may lead to cumbersome integrals to evaluate.

${ }^{9}$ This approximation is not made in developing our general theory, but just in obtaining specific illustrations.
}

As in I, we represent the counting rate of detector " 1 " by the counting-rate operator, at time $T_{1}$,

$$
\begin{aligned}
G_{1} \equiv \int_{-\infty}^{+\infty} d t_{1} L_{1}\left(T_{1}-t_{1}\right) & \int_{1} d^{3} y_{1} \gamma\left(\mathbf{y}_{1}\right) \\
& \times \sum_{l=1}^{n} e^{i K_{l t_{1}}} \delta\left(\mathbf{y}_{1}-\mathbf{x}_{l}\right) e^{-i K_{l} t_{1}} .
\end{aligned}
$$

Here $K_{l}$ is the kinetic-energy operator for the $l$ th beam particle, so $\exp \left[i K_{l} t_{1}\right] \mathbf{x}_{l} \exp \left[-i K_{l} t_{1}\right]$ is its position vector in the Heisenberg representation. The function $\gamma_{1}\left(\mathbf{y}_{1}\right)$ is introduced to take account of the detector calibration. The integration over $\mathbf{y}_{1}$ in (2.10) extends over the active volume of the detector. The function $L_{1}(\tau)$ is introduced to represent the detector's transient response characteristics.

For a uniform detector having $100 \%$ efficiency and calibrated to measure the total beam flux striking the area $\Sigma_{1}$, we evidently have

$$
\gamma_{1}=V / w_{1}
$$

Since the gain setting on the electronics is irrelevant, as long as the detector has been calibrated, we shall assume (without loss of generality) the calibration (2.11). We shall then take account of the actual detector efficiency by supposing that the fluxes of particles appearing in our expressions represent measured, rather than actual fluxes. That is, we shall formally treat our detectors as having unit efficiency and later correct for limited efficiency by a reinterpretation of our calculated fluxes.

Continuing to follow the notation of I, we give the transient response function $L_{1}(\tau)$ the Fourier integral representation

$$
L_{1}(\tau)=\int \frac{d \Omega}{2 \pi} B_{1}(\Omega) e^{-i \Omega \tau} .
$$

A transient response time $\Delta \tau_{r}$ for the detector is defined by the equation

$$
\frac{1}{\Delta \tau_{r}} \equiv \int \frac{d \Omega}{2 \pi}\left|B_{1}(\Omega)\right|^{2} .
$$

The mean counting rate during the experiment is

$$
\left\langle G_{1}\right\rangle=\left\langle\left(\Psi(0), G_{1}\left(T_{1}\right) \Psi(0)\right)\right\rangle .
$$

Here the symbol " $\langle\cdots\rangle$ " on the right represents an ensemble average over many observations. By hypothesis, $\left\langle G_{1}\right\rangle$ is independent of the time $T_{1}$ in the interval $T$.

For the "calibrated detector" defined by Eq. (2.11) we have ${ }^{10}$

$$
\left\langle G_{1}\right\rangle=B_{1}(0) \Sigma_{1} F\left(\mathbf{Y}_{1}\right)
$$

${ }^{10}$ Recalling the remarks made in connection with Eq. (2.11), we shall eventually insert a numerical factor into the right side of Eq. (2.15) to take account of the detector efficiency. 
To be consistent with Eq. (2.11) we shall always assume either that

or

$$
B_{1}(0)=1 \text {, }
$$

$$
B_{1}(0)=0 \text {, }
$$

corresponding to a dc blocking filter being placed in the detector output. The flux corresponding to $B_{1}(0)=1$ will be denoted as $\left\langle G_{1}\right\rangle_{0}$, that is,

$$
\left\langle G_{1}\right\rangle_{0} \equiv\left\langle G_{1}\right\rangle / B_{1}(0) \text {. }
$$

An explicit evaluation of $\left\langle G_{1}\right\rangle$, Eq. (2.14), was given in $\mathrm{I}$ in terms of the beam wave function, $\mathrm{Eq}$. (2.1), where we found

$$
\left\langle G_{1}\right\rangle=B_{1}(0) \Sigma_{1} w_{1} \gamma_{1} \bar{n} \chi(1),
$$

where $\bar{n}=\langle n\rangle$ is the mean number of particles emitted in the interval $T$, and

$$
\begin{aligned}
\chi(1) & \equiv\left\langle\Phi_{j}^{*}\left(\mathbf{y}_{1}, t_{1}\right) \Phi_{j}\left(\mathbf{y}_{1}, t_{1}\right)\right\rangle \\
& =N_{B} / \bar{n} y_{1}{ }^{2} .
\end{aligned}
$$

Here $N_{B}$ is a constant, seen from Eqs. (2.2) and (2.15) to be

$$
N_{B}=R_{B} / 4 \pi V \text {. }
$$

To take account of the actual efficiency $\eta$ of the detectors we shall replace Eq. (2.20a) by

$$
N_{B}=\eta\left(R_{B} / 4 \pi V\right) \text {. }
$$

The detector thickness $w_{1}$ must be defined a little carefully. If the particles are stopped in a distance small compared to the actual physical thickness, we should interpret $w_{1}$ as the mean range of the stopped particles. The principal significance of $w_{1}$ is that $w_{1} / V$ represents a spread in arrival times. If machining accuracy and detector alignment errors lead to an effective $w_{1} / V$ larger than that associated with the stopping distance, we determine $w_{1}$ from these.

To describe a particle correlation experiment we introduce a second detector, " 2 ," as in Fig. 2, located at $\mathbf{Y}_{2}$. The outputs of the two detectors are multiplied together in a correlator, after passing one of them through a delay line having a delay time $\tau$. The output of the correlator is represented by the operator

$$
G_{12}\left(T_{2}, T_{1}\right)=G_{2}\left(T_{2}\right) G_{1}\left(T_{1}\right),
$$

where $T_{2}=T_{1}+\tau$ and $G_{2}$ is described by an expression like Eq. (2.10) for $G_{1}$ but with subscript 1 replaced by 2 . We are tacitly assuming that the situations of interest

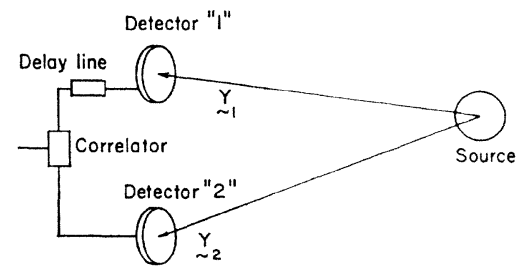

Fig. 2. An intensity correlation experiment.

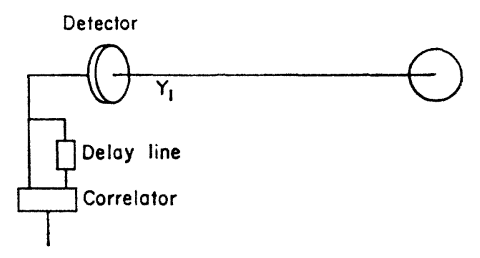

Fig. 3. A measurement of the autocorrelation function.

are such that $G_{1}$ and $G_{2}$ effectively commute; otherwise, as discussed in I, the operator (2.21) is not the appropriate one. For example, it is not generally Hermitian. For our subsequent estimates, it will be convenient to suppose that the two detectors have similar characteristics, setting

$$
\begin{aligned}
& \Sigma_{1}=\Sigma_{2}=\Sigma_{D} \\
& w_{1}=w_{2}=w \\
& B_{1}=B_{2}=B
\end{aligned}
$$

etc.

The ensemble average of the correlator output over the interval $T$ is

$$
\left\langle G_{12}\right\rangle=\left\langle\left(\Psi(0), G_{12} \Psi(0)\right)\right\rangle .
$$

This in general depends on the time delay $\tau=T_{2}-T_{1}$ and not on $T_{1}$ or $T_{2}$ individually.

An experiment designed to study correlations with a single counter is illustrated in Fig. 3. The direct output and the output passed through the delay line are again mixed in the correlator. The correlator output here is also described by Eq. (2.23) if we imagine letting the two detectors of Fig. 2 coalesce into a single counter at $\mathbf{Y}_{1}$.

In this paper we consider only the case for which the two counters are "not in line," so a single particle cannot give a count in both of them. This condition is automatically satisfied if the detected particles are stopped or absorbed in the detectors or if, in fact, there is only a single detector. Then, the evaluation of (2.23) given in I leads to the result (the + and - signs refer to the cases of Bose-Einstein and Fermi-Dirac statistics, respectively)

$$
\left\langle G_{12}\right\rangle=\left\langle G_{1}\right\rangle\left\langle G_{2}\right\rangle \pm \bar{n}^{2} g \int(1) \int(2)|\chi(12)|^{2},
$$

where $g$ is the spin-average factor [see Eq. (2.38) of $\mathrm{I}]$. Here we have introduced the abbreviations

$$
\int(1) \cdots=\int d t_{1} L_{1}\left(T_{1}-t_{1}\right) \int_{1} d^{3} y_{1} \gamma_{1}\left(\mathbf{y}_{1}\right) \cdots,
$$

etc., and [see Eq. (3.17) of I]

where

$$
\chi(12)=\chi_{p}(12) Q(12)
$$

$\chi_{p}(12)=\frac{N_{B}}{\bar{n} y_{1} y_{2}} \int d \omega g(\omega)$

$$
\times \exp \left\{i\left[q\left(y_{2}-y_{1}\right)-\omega\left(t_{2}-t_{1}\right)\right]\right\},
$$


and

$$
Q(12)=\int_{s} \frac{d^{3} s}{\nu_{s}} \exp \left[i p\left(\hat{y}_{1}-\hat{y}_{2}\right) \cdot \mathbf{s}\right]
$$

The quantity $q$ in Eq. (2.26b) is the momentum (wave number) of a beam particle having energy $\hbar \omega$. In Eq. (2.26c), $V_{8}$ is the source volume and the integral extends over all source points s; also, the wave number has been set equal to the central value $p$.

We note that for a sufficiently small source $Q(12) \cong 1$ and $\chi$ may be replaced by $\chi_{p}$. We also note that if the beam flux is not the same at the two detectors we must write $N_{B}(1), R_{B}(1), N_{B}(2), R_{B}(2)$, etc., to describe the respective intensities at " 1 " and " 2. ."

It is the quantity

$$
\left\langle\Delta G_{12}\right\rangle \equiv\left\langle G_{12}\right\rangle-\left\langle G_{1}\right\rangle\left\langle G_{2}\right\rangle
$$

which is of physical interest for fluctuation and correlation experiments. It is possible to measure $\left\langle\Delta G_{12}\right\rangle$ directly by placing dc blocking filters in the outputs of the two detectors, so $\left\langle G_{1}\right\rangle=\left\langle G_{2}\right\rangle=0$ [see Eqs. (2.15) and (2.16b)]. Then

$$
\left\langle\Delta G_{12}\right\rangle=\left\langle G_{12}\right\rangle .
$$

For a small point source for which $\chi(12) \cong \chi_{p}(12)$, we find for $\left\langle\Delta G_{12}\right\rangle$ [see Eq. (3.9) of I], using Eq. (2.24),

$$
\begin{aligned}
& \left\langle\Delta G_{12}\right\rangle=\left\langle\Delta G_{12}\right\rangle_{p}= \pm\left\langle G_{1}\right\rangle_{0}\left\langle G_{2}\right\rangle_{0} g \\
& \quad \times \int_{1} \frac{d^{3} y_{1}}{\Sigma_{1} w_{1}} \int_{2} \frac{d^{3} y_{2}}{\Sigma_{2} w_{2}} d \omega d \omega^{\prime} g(\omega) g\left(\omega^{\prime}\right) B_{1}\left(\omega^{\prime}-\omega\right) B_{2}\left(\omega-\omega^{\prime}\right) \\
& \quad \times \exp \left\{i\left[\left(q-q^{\prime}\right)\left(y_{2}-y_{1}\right)-\left(\omega-\omega^{\prime}\right)\left(T_{2}-T_{1}\right)\right]\right\} .
\end{aligned}
$$

For a finite source we must keep $Q(12)$ in Eq. (2.26a). If the detectors are well enough aligned with respect to the beam we may evaluate (2.24) in the form

$$
\left\langle\Delta G_{12}\right\rangle=\left\langle\Delta G_{12}\right\rangle_{p} I_{S},
$$

where

$$
I_{S} \equiv \int_{1} \frac{d^{2} v_{1}}{\Sigma_{1}} \int_{2} \frac{d^{2} v_{2}}{\Sigma_{2}}|Q(12)|^{2}
$$

Here $\mathbf{v}_{1}$ is the projection of $\mathbf{u}_{1} \equiv \mathbf{y}_{1}-\mathbf{Y}_{1}$ on a plane perpendicular to $\mathbf{Y}_{1}$, etc., $I_{S}$ is a function of the dimensionless quantity

$$
\sigma \equiv Y^{2} \lambda^{2} / \Sigma_{s} \Sigma_{D},
$$

where $\Sigma_{s}$ and $\Sigma_{D}$ are, respectively, the source and detector areas, $Y \sim Y_{1} \sim Y_{2}$ is the distance from the source to the detectors, and $\lambda=2 \pi / p$ is the particle de Broglie wavelength. An evaluation of $I_{S}$ gives

$$
\begin{aligned}
& I_{S}=1, \quad \text { for } \sigma \gg 1 \text {, } \\
& =Y^{2} \lambda^{2} / \Sigma_{s} \Sigma_{D}, \text { for } \sigma \ll 1 \text {. }
\end{aligned}
$$

A comparison with such descriptions as those given by Purcell ${ }^{11}$ and by Twiss and Little ${ }^{12}$ may be convenient at this point. They discuss the number of coincidences $N_{c}$ between pairs of particles during the time $T$. This may be done most easily when the expected number of particle arrivals in the resolving-time interval $\Delta \tau_{r}$ is much less than unity. Then (with a little more attention given to the definition of $\left.\Delta \tau_{r}\right)$

$$
\left\langle N_{c}\right\rangle=\Delta \tau_{r} T\left\langle G_{12}\right\rangle \text {. }
$$

The term $\Delta \tau_{r} T\left\langle G_{1}\right\rangle\left\langle G_{2}\right\rangle$ has been described ${ }^{12}$ as due to "random coincidences" and $\Delta \tau_{r} T\left\langle\Delta G_{12}\right\rangle$ as due to particle "clumping." This kind of description is picturesque, but of limited applicability (as, for example, to the case of electron beams or to the case in which many particles are counted during one resolving time $\Delta \tau_{r}$ ).

Our results may be applied to a scattering experiment, as explained in I. Such an experiment is illustrated in Fig. 4. It is necessary only to include appropriate scattering amplitudes and fluxes incident on the target in the normalization constants $N_{B}(1)$ and $N_{B}(2)$. In this case the target plays the role of the "source" in the discussion given above.

\section{ACCURACY OF A SINGLE COUNTING EXPERIMENT}

We begin our study of measurement accuracy by considering a simple counting experiment, as is illustrated in Fig. 1. The total number of counts in the interval $T$ is, on the average,

$$
\left\langle N_{T}\right\rangle \equiv\left\langle\int_{0}^{T} d T_{1} G_{1}\right\rangle=T\left\langle G_{1}\right\rangle,
$$

where $\left\langle G_{1}\right\rangle$ is given by Eq. (2.15) and we now assume that $B_{1}(0)=1$. The fluctuations in the number of counts may be expressed in terms $\left\langle N_{T^{2}}{ }^{2}\right.$, where

$$
\begin{aligned}
\left\langle N_{T^{2}}{ }^{2}\right\rangle=\left\langle\left[\int_{0}^{T} d T_{1} G_{1}\right]^{2}\right. & \rangle \\
& =\left\langle\int_{0}^{T} d T_{1} \int_{0}^{T} d T_{2} G_{12}\right\rangle .
\end{aligned}
$$

In the last step here we think of the two detectors as being coalesced into one, as described in connection with Eq. (2.21).

From the explicit form of the counting operator $G_{1}$,

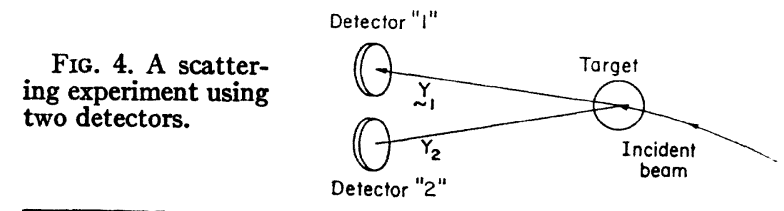

11 E. M. Purcell, Nature 178, 1449 (1956).

12 R. Q. Twiss and A. G. Little, Australian J. Phys. 12,77 (1959). 
Eqs. (2.10) and (2.21), we obtain

$$
\begin{aligned}
&\left\langle N_{T^{2}}\right\rangle=\int_{0}^{T} d T_{1} \int_{0}^{T} d T_{2} \int(1) \int(2)\left\langle\left(\Psi(0),\left\{\sum_{k \neq l} e^{i K_{k} t_{2}} \delta\left(\mathbf{y}_{2}-\mathbf{x}_{1}\right) e^{-i K_{k} t_{2}} e^{i K_{l} t_{1}} \delta\left(\mathbf{y}_{1}-\mathbf{x}_{i}\right) e^{-i K_{l} t_{1}}\right.\right.\right. \\
&\left.\left.\left.+\sum_{l} e^{i K_{l} t_{2}} \delta\left(\mathbf{y}_{2}-\mathbf{x}_{l}\right) e^{-i K_{l}\left(t_{2}-t_{1}\right)} \delta\left(\mathbf{y}_{1}-\mathbf{x}_{l}\right) e^{-i K_{l} t_{1}}\right\} \Psi(0)\right)\right\rangle .
\end{aligned}
$$

Consider first the "nondiagonal terms," corresponding to $l \neq k$ in the sum above. These are just the terms evaluated in Eq. (2.24). Thus, on comparing Eqs. (2.24) and (3.2), we have for the nondiagonal contribution $\left\langle N_{T^{2}}\right\rangle_{n}$,

$$
\begin{aligned}
\left\langle N_{T}{ }^{2}\right\rangle_{n}=\int_{0}^{T} d & T_{1} \int_{0}^{T} d T_{2}\left\{\left\langle G_{1}\right\rangle\left\langle G_{2}\right\rangle\right. \\
& \left. \pm \bar{n}^{2} g \int(1) \int(2)|\chi(12)|^{2}\right\} \\
& =\left[\left\langle N_{T}\right\rangle\right]^{2} \pm \int_{0}^{T} d T_{1} \int_{0}^{T} d T_{2}\left\langle\Delta G_{12}\right\rangle .
\end{aligned}
$$

[We have set $\left\langle G_{2}\right\rangle=\left\langle G_{1}\right\rangle$, since we are considering only a single detector.] On using Eqs. (2.29) and (2.30) here and recalling that $T$ is very long compared to any transient time scales (such as $1 / \Delta \omega_{B}$ and $\Delta \tau_{r}$ ), we find

$$
\left\langle V_{T^{2}}\right\rangle_{n}=\left[\left\langle N_{T}\right\rangle\right]^{2} \pm 2 \pi g I_{s}\left(\left\langle G_{1}\right\rangle / \Delta \omega_{B}\right)\left\langle N_{T}\right\rangle .
$$

To evaluate the diagonal terms in Eq. (3.3) we use the approximate relation

$$
\begin{aligned}
e^{i K_{l \tau} \delta}\left(\mathbf{x}_{l}-\mathbf{y}_{1}\right) e^{-i K_{l \tau} \delta}\left(\mathbf{x}_{l}-\mathbf{y}_{2}\right) & \\
& =\delta\left(\mathbf{y}_{2}-\mathbf{y}_{1}+\tau V \hat{y}_{2}\right) \delta\left(\mathbf{x}_{l}-\mathbf{y}_{2}\right),
\end{aligned}
$$

valid because of our assumed narrow beam spectrum. ${ }^{13}$

The diagonal contribution then becomes

$$
\begin{aligned}
\left\langle N_{T^{2}}\right\rangle_{d}=\bar{n} \int_{0}^{T} d T_{1} \int_{0}^{T} d T_{2} & \\
& \times \int(1) \int(2) \delta\left(\mathbf{y}_{2}-\mathbf{y}_{1}+\tau V \hat{y}_{2}\right) \chi(2) \\
= & T\left\langle G_{1}\right\rangle=\left\langle N_{T}\right\rangle .
\end{aligned}
$$

On combining Eqs. (3.5) and (3.7), we obtain

$$
\left\langle N_{T^{2}}{ }^{2}\right\rangle=\left[\left\langle N_{T}\right\rangle\right]^{2}+\left\langle N_{T}\right\rangle 1 \pm 2 \pi g I_{s}\left(\left\langle G_{1}\right\rangle / \Delta \omega_{B}\right) .
$$

Under most practical experimental conditions

$$
2 \pi g I_{s}\left(\left\langle G_{1}\right\rangle / \Delta \omega_{B}\right) \ll 1,
$$

${ }^{13}$ In obtaining Eq. (3.6) we have used the nonrelativistic relation

$$
\exp \left[i K_{l} \tau\right] \mathbf{x}_{l} \exp \left[-i K_{l} \tau\right]=\mathbf{x}_{l}+\tau(\mathbf{q} / M),
$$

where $q$ is the momentum operator and $M$ is the mass of a beam particle. Because of the assumed narrow beam spectrum, we set $\mathrm{q} / M=V$. [See Eq. (5.7) of I for a more general result.] The result (3.6) jay also be derived for relativistic particles by writing $K_{l}(\mathbf{q}) \cong K_{l}(\mathbf{p})+\mathbf{V} \cdot(\mathbf{q}-\mathbf{p})$ and Eq. (3.8) reduces to the "classical" expression

$$
\left\langle N_{T^{2}}{ }^{2} \cong\left[\left\langle N_{T}\right\rangle\right]^{2}+\left\langle N_{T}\right\rangle .\right.
$$

For a point source, $I_{s} \approx 1$. It can be shown that for a degenerate beam of Fermi-Dirac particles, the maximum value of $\left\langle G_{1}\right\rangle$ corresponds to $\Delta \omega_{B} / 2 \pi g$, so that for a point source

$$
\left\langle N_{T}{ }^{2}\right\rangle \cong\left\langle N_{T}\right\rangle^{2}
$$

and the fluctuations are significantly reduced. This maximum value for the quantum-mechanical term in Eq. (3.8) does not appear in a natural way since we have not explicitly allowed for having used up all available states in the evaluation of expectation values with the wave function (2.1).

\section{FLUCTUATIONS IN INTENSITY CORRELATION EXPERIMENTS}

We turn now to a study of the accuracy of measurements of intensity correlations of the variety implied by the expression Eq. (2.23) for the expectation value of the product of two counting operators. We are thus concerned with the description of Hanbury-Brown and Twiss particle correlations in two counters, interference effects in a single counter, ${ }^{4}$ and beam (or target) fluctuations., ${ }^{1,6}$ We may reduce the latter two classes to a special case of the Hanbury-Brown and Twiss variety by imagining that the two counters are combined into one.

We continue to suppose that the experimental situation is such that the two counters are not in line in the sense that a single particle cannot give a count in each of them. This is the same assumption that we made previously in our discussion of correlation experiments [see Eq. (2.24)]. Then in Eq. (2.21) we set $T_{2}=T_{1}+\tau$ and from the "average" quantity

$$
\begin{aligned}
G_{\mathrm{av}}(\tau) \equiv \int_{0}^{T} d T_{1} G_{12}\left(T_{1}, T_{1}+\tau\right) \\
=\int_{0}^{T} d T_{1} G_{1}\left(T_{1}\right) G_{2}\left(T_{1}+\tau\right) .
\end{aligned}
$$

Evidently, by our assumption that the ensembleaveraged beam intensity is uniform throughout the interval $T$, we have

$$
\left\langle G_{\mathrm{av}}(\tau)\right\rangle \equiv\left\langle\left(\Psi(0), G_{\mathrm{av}} \Psi(0)\right)\right\rangle=T\left\langle G_{12}(\tau)\right\rangle .
$$


To study the fluctuations over the ensemble of the measured values of $G_{\mathrm{av}}$, we must evaluate the quantity

$$
\left\langle G_{\mathrm{av}}{ }^{2}(\tau)\right\rangle=\left\langle\left(\Psi(0), G_{\mathrm{av}}{ }^{2}(\tau) \Psi(0)\right)\right\rangle .
$$

To evaluate this expression we first substitute the appropriate expression (2.10) into Eq. (4.1) and then into (4.3). Making use of our assumption that a given particle cannot pass on a straight line through both counters, we obtain

$$
\begin{aligned}
\left\langle G_{\mathrm{av}}{ }^{2}\right\rangle=K\left\langle\left(\Psi(0), \sum_{l \neq k=1}^{n} \sum_{m \neq s=1}^{n} \delta\left[\mathbf{x}_{l}\left(t_{1}\right)-\mathbf{y}_{1}\right]\right.\right. \\
\times \delta\left[\mathbf{x}_{k}\left(t_{2}\right)-\mathbf{y}_{2}\right] \delta\left[\mathbf{x}_{m}\left(t_{1}^{\prime}\right)-\mathbf{y}_{1}{ }^{\prime}\right] \\
\left.\left.\times \delta\left[\mathbf{x}_{8}\left(t_{2}{ }^{\prime}\right)-\mathbf{y}_{2}{ }^{\prime}\right] \Psi(0)\right)\right\rangle .
\end{aligned}
$$

Here $K$ is the integral operator

$$
\begin{aligned}
& K \cdots=\int_{0}^{T} d T_{1} \int_{0}^{T} d T_{1} \int_{0}^{T} d t_{1} \int_{0}^{T} d t_{2} \int_{0}^{T} d t_{1} \int_{0}^{T} d t_{2}^{\prime} L_{1}\left(T_{1}-t_{1}\right) L_{2}\left(T_{1}+\tau-t_{2}\right) \\
& \times L_{1}\left(T_{1}^{\prime}-t_{1}^{\prime}\right) L_{2}\left(T_{1}^{\prime}+\tau-t_{2}{ }^{\prime}\right) \gamma_{1}{ }^{2} \gamma_{2}{ }^{2} \int_{1} d^{3} y_{1} \int_{1} d^{3} y_{1} \int^{\prime} d^{3} y_{2} \int_{2} d^{3} y_{2}{ }^{\prime} \cdots \\
& =\gamma_{1}^{2} \gamma_{2}{ }^{2} \int_{1} d^{2} y_{1} \int_{1} d^{3} y_{1} \int_{2}^{\prime} d^{3} y_{2} \int_{2} d^{3} y_{2}^{\prime} \int_{0}^{T} d t_{1} \int_{0}^{T} d t_{1}^{\prime} \int_{0}^{T} d t_{2} \int_{0}^{T} d t_{2}^{\prime} \\
& \times \int \frac{d \Omega}{2 \pi} B_{1}(-\Omega) B_{2}(\Omega) e^{i \Omega\left(t_{2}-t_{1}-\tau\right)} \int \frac{d \Omega^{\prime}}{2 \pi} B_{1}\left(\Omega^{\prime}\right) B_{2}\left(-\Omega^{\prime}\right) e^{i \Omega^{\prime}\left(t_{1}^{\prime}-t 2^{\prime}+\tau\right)} \ldots .
\end{aligned}
$$

In arriving at the second form of $K$ we have imagined that the interval $T$ is very long compared with any transient times. Thus having expressed the filter functions $L_{i}\left(T_{1}-t_{i}\right)$ in terms of the frequency response according to Eq. (2.12) we may carry out the integration over $T_{1}, T_{1}{ }^{\prime}$ and obtain $\delta$ functions. It is also a matter of indifference whether we regard the $t_{2}, t_{1} \cdots$ integration limits to be $-\infty$ to $\infty$ or 0 to $T$.

It is important to remember that the quantities $\mathbf{x}_{l}\left(t_{1}\right)$ are the rather complicated Heisenberg variables

$$
\mathbf{x}_{l}\left(t_{1}\right)=e^{i K l t_{1}} \mathbf{x}_{l} e^{-i K i t_{1}} .
$$

Further, we note that the restrictions $k \neq l, s \neq m$ in Eq. (4.4) follow from our demand that the same particle cannot pass through both counters. In the summand in Eq. (4.4), we may have index pairings $l=m$ and/or $k=s$, but we may not have $l=s$ or $k=m$; this would again require that a particle be countable in both detectors. This suggests grouping the terms according to whether there are two, three, or four unequal indices. We write then

$$
\left\langle G_{\mathrm{av}}{ }^{2}\right\rangle=\Gamma_{2}+\Gamma_{3}(1)+\Gamma_{3}(2)+\Gamma_{4},
$$

where

$$
\begin{aligned}
\Gamma_{2}=K\langle & \left(\Psi(0), \sum_{l \neq k} \delta\left(\mathbf{x}_{l}\left(t_{1}\right)-\mathbf{y}_{1}\right) \delta\left(\mathbf{x}_{l}\left(t_{1}{ }^{\prime}\right)-\mathbf{y}_{1}{ }^{\prime}\right)\right. \\
& \left.\left.\times \delta\left(\mathbf{x}_{k}\left(t_{2}\right)-\mathbf{y}_{2}\right) \delta\left(\mathbf{x}_{k}\left(t_{2}{ }^{\prime}\right)-\mathbf{y}_{2}{ }^{\prime}\right) \Psi(0)\right)\right\rangle,
\end{aligned}
$$

$$
\begin{aligned}
& \Gamma_{3}(1)=K\left\langle\left(\Psi(0), \sum_{l \neq k \neq \neq} \delta\left(\mathbf{x}_{l}\left(t_{1}\right)-\mathbf{y}_{\mathbf{1}}\right) \delta\left(\mathbf{x}_{l}\left(t_{1}{ }^{\prime}\right)-\mathbf{y}_{1}{ }^{\prime}\right)\right.\right. \\
& \left.\left.\times \delta\left(\mathbf{x}_{k}\left(t_{2}\right)-\mathbf{y}_{2}\right) \delta\left(\mathbf{x}_{\mathbf{s}}\left(t_{2}{ }^{\prime}\right)-\mathbf{y}_{2}{ }^{\prime}\right) \Psi(0)\right)\right\rangle, \\
& \Gamma_{3}(2)=K\left\langle\left(\Psi(0), \sum_{l \neq m \neq k} \delta\left(\mathbf{x}_{l}\left(t_{1}\right)-\mathbf{y}_{1}\right) \delta\left(\mathbf{x}_{m}\left(t_{1}{ }^{\prime}\right)-\mathbf{y}_{\mathbf{1}}{ }^{\prime}\right)\right.\right. \\
& \left.\times \delta\left(\mathbf{x}_{k}\left(t_{2}\right)-\mathbf{y}_{2}\right) \delta\left(\mathbf{x}_{k}\left(t_{2}^{\prime}-\mathbf{y}_{2}^{\prime}\right) \Psi(0)\right)\right\rangle, \\
& \Gamma_{4}=K\left\langle\left(\Psi(0), \sum_{l \neq m \neq k \neq s} \delta\left(\mathbf{x}_{l}\left(t_{1}\right)-\mathbf{y}_{1}\right) \delta\left(\mathbf{x}_{m}\left(t_{1}^{\prime}\right)-\mathbf{y}_{1}{ }^{\prime}\right)\right.\right. \\
& \left.\left.\times \delta\left(\mathbf{x}_{k}\left(t_{2}\right)-\mathbf{y}_{2}\right) \delta\left(\mathbf{x}_{\boldsymbol{s}}\left(t_{2}{ }^{\prime}\right)-\mathbf{y}_{2}{ }^{\prime}\right) \Psi(0)\right)\right\rangle .
\end{aligned}
$$

The notation here is meant to imply that in no one of the four $\Gamma$ 's can two of the indices $k, l, m, s$ be equal. Thus, the interference of two particles is described by $\Gamma_{2}$, the interference of three particles by $\Gamma_{3}(1)$ and $\Gamma_{3}(2)$, and the interference of four particles by $\Gamma_{4}$. We note that having evaluated $\Gamma_{3}(1)$, we can obtain $\Gamma_{3}(2)$ from this by interchanging the detector labels " 1 " and "2" and changing the sign of $\tau$.

The expressions for $\Gamma_{2}$ and $\Gamma_{3}$ may be simplified if we make use of the previously given relation for the product of $\delta$ functions involving the same operators at different times, Eq. (3.6), which we utilize in the form

$$
\begin{aligned}
& \delta\left[\mathbf{y}_{\mathbf{1}}-\mathbf{x}_{l}\left(t_{1}\right)\right] \delta\left[\mathbf{y}_{1}{ }^{\prime}-\mathbf{x}_{l}\left(t_{1}{ }^{\prime}\right)\right]=e^{i K l t_{1}{ }^{\prime}} e^{i K l r_{1}} \delta\left(\mathbf{y}_{1}-\mathbf{x}_{l}\right) e^{-i K l r_{1}} \delta\left(\mathbf{y}_{1}{ }^{\prime}-\mathbf{x}_{l}\right) e^{-i K l t_{1}{ }^{\prime}} \\
& =\delta\left(\mathbf{y}_{1}{ }^{\prime}-\mathbf{y}_{1}+\tau_{1} V^{\prime} \hat{y}_{1}^{\prime}\right) e^{i K l t_{1}^{\prime}} \delta\left(\mathbf{y}_{1}{ }^{\prime}-\mathbf{x}_{l}\right) e^{-i K l t_{1}{ }^{\prime}},
\end{aligned}
$$

where $\tau_{1}=t_{1}-t_{1}{ }^{\prime}$ and we shall also need $\tau_{2}=t_{2}-t_{2}{ }^{\prime}$. We find then

$$
\begin{aligned}
\Gamma_{2}=K\left\{\delta\left(\mathbf{y}_{1}{ }^{\prime}-\mathbf{y}_{1}+\tau_{1} V \hat{y}_{1}{ }^{\prime}\right) \delta\left(\mathbf{y}_{2}{ }^{\prime}-\mathbf{y}_{2}+\tau_{2} V \hat{y}_{2}{ }^{\prime}\right)\langle(\Psi(0)\right. & \sum_{l \neq k} \exp \left[i\left(K_{l} t_{1}{ }^{\prime}+K_{k} t_{2}{ }^{\prime}\right)\right] \\
& \left.\left.\left.\times \delta\left(\mathbf{x}_{l}-\mathbf{y}_{1}{ }^{\prime}\right) \delta\left(\mathbf{x}_{k}-\mathbf{y}_{2}{ }^{\prime}\right) \exp \left[-i\left(K_{l} t_{1}{ }^{\prime}+K_{k} t_{2}{ }^{\prime}\right)\right] \Psi(0)\right)\right\rangle\right\}
\end{aligned}
$$




$$
\begin{aligned}
\Gamma_{3}(1)=K\left\{\delta\left(\mathbf{y}_{1}{ }^{\prime}-\mathbf{y}_{1}+\tau_{1} V \hat{y}_{1}\right)\langle(\Psi(0),\right. & \sum_{l \neq k \neq \neq} \exp \left[i\left(K_{l} t_{1}{ }^{\prime}+K_{k} t_{2}+K_{\mathrm{e}} t_{2}{ }^{\prime}\right)\right] \\
& \left.\left.\left.\times \delta\left(\mathbf{x}_{l}-\mathbf{y}_{1}{ }^{\prime}\right) \delta\left(\mathbf{x}_{k}-\mathbf{y}_{2}\right) \delta\left(\mathbf{x}_{\mathbf{s}}-\mathbf{y}_{2}{ }^{\prime}\right) \exp \left[-i\left(K_{l} t_{1}{ }^{\prime}+K_{k} t_{2}+K_{8} t_{2}{ }^{\prime}\right)\right] \Psi(0)\right)\right\rangle\right\},
\end{aligned}
$$

etc.

A straightforward but tedious calculation using the wave function (2.1) gives, finally, ${ }^{14}$

$$
\begin{gathered}
\Gamma_{2}=\bar{n}^{2} K\left\{\delta\left(\mathbf{y}_{1}^{\prime}-\mathbf{y}_{1}+V \tau_{1} \hat{y}_{1}^{\prime}\right) \delta\left(\mathbf{y}_{2}{ }^{\prime}-\mathbf{y}_{2}+V \tau_{2} \hat{y}_{2}^{\prime}\right)\left[\chi\left(1^{\prime}\right) \chi\left(2^{\prime}\right) \pm\left|\chi\left(1^{\prime} 2^{\prime}\right)\right|^{2}\right]\right\} \\
\Gamma_{3}(1)=\bar{n}^{3} K\left\{\delta ( \mathbf { y } _ { 1 } { } ^ { \prime } - \mathbf { y } _ { 1 } + V \tau _ { 1 } \hat { y } _ { 1 } ) \left[\chi\left(1^{\prime}\right) \chi(2) \chi\left(2^{\prime}\right) \pm\left|\chi\left(1^{\prime} 2\right)\right|^{2} \chi\left(2^{\prime}\right) \pm \chi\left(1^{\prime}\right)\left|\chi\left(22^{\prime}\right)\right|^{2}\right.\right. \\
\left.\left. \pm \chi(2)\left|\chi\left(1^{\prime} 2^{\prime}\right)\right|^{2}+\chi\left(1^{\prime} 2\right) \chi\left(22^{\prime}\right) \chi\left(2^{\prime} 1^{\prime}\right)+\chi\left(1^{\prime} 2^{\prime}\right) \chi\left(2^{\prime} 2\right) \chi\left(21^{\prime}\right)\right]\right\} \\
\Gamma_{3}(2)= \\
n^{3} K\left\{\delta ( \mathbf { y } _ { 2 } { } ^ { \prime } - \mathbf { y } _ { 2 } + V \tau _ { 2 } \hat { y } _ { 2 } ) \left[\chi\left(2^{\prime}\right) \chi(1) \chi\left(1^{\prime}\right) \pm\left|\chi\left(2^{\prime} 1\right)\right|^{2} \chi\left(1^{\prime}\right)\right.\right. \\
\left.\left. \pm \chi\left(2^{\prime}\right)\left|\chi\left(11^{\prime}\right)\right|^{2} \pm \chi(1)\left|\chi\left(2^{\prime} 1^{\prime}\right)\right|^{2}+\chi\left(2^{\prime} 1\right) \chi\left(11^{\prime}\right) \chi\left(1^{\prime} 2^{\prime}\right)+\chi\left(2^{\prime} 1^{\prime}\right) \chi\left(1^{\prime} 1\right) \chi\left(12^{\prime}\right)\right]\right\}
\end{gathered}
$$

and

$$
\begin{aligned}
\Gamma_{4}= & {\left[\left\langle G_{\mathrm{av}}(\tau)\right\rangle\right]^{2}+\bar{n}^{4} K\left\{ \pm \chi(1) \chi\left(1^{\prime} 2\right) \chi\left(21^{\prime}\right) \chi\left(2^{\prime}\right)\right.} \\
& +\chi(1) \chi\left(2^{\prime} 2\right) \chi\left(21^{\prime}\right) \chi\left(1^{\prime} 2^{\prime}\right) \pm \chi(1) \chi\left(2^{\prime} 2\right) \chi\left(1^{\prime}\right) \chi\left(22^{\prime}\right)+\chi(1) \chi\left(1^{\prime} 2\right) \chi\left(2^{\prime} 1^{\prime}\right) \chi\left(22^{\prime}\right)+\chi(21) \chi\left(1^{\prime} 2\right) \chi\left(11^{\prime}\right) \chi\left(2^{\prime}\right) \\
& \pm \chi(21) \chi\left(2^{\prime} 2\right) \chi\left(11^{\prime}\right) \chi\left(1^{\prime} 2^{\prime}\right)+\chi(21) \chi\left(2^{\prime} 2\right) \chi\left(1^{\prime}\right) \chi\left(12^{\prime}\right) \pm \chi(21) \chi\left(1^{\prime} 2\right) \chi\left(2^{\prime} 1^{\prime}\right) \chi\left(12^{\prime}\right) \pm \chi\left(1^{\prime} 1\right) \chi(2) \chi\left(11^{\prime}\right) \chi\left(2^{\prime}\right) \\
& +\chi\left(2^{\prime} 1\right) \chi(2) \chi\left(11^{\prime}\right) \chi\left(1^{\prime} 2^{\prime}\right) \pm \chi\left(2^{\prime} 1\right) \chi(2) \chi\left(1^{\prime}\right) \chi\left(12^{\prime}\right)+\chi\left(1^{\prime} 1\right) \chi(2) \chi\left(2^{\prime} 1^{\prime}\right) \chi\left(12^{\prime}\right)+\chi\left(1^{\prime} 1\right) \chi(12) \chi\left(21^{\prime}\right) \chi\left(2^{\prime}\right) \\
& \pm \chi\left(2^{\prime} 1\right) \chi(12) \chi\left(21^{\prime}\right) \chi\left(1^{\prime} 2^{\prime}\right)+\chi\left(2^{\prime} 1\right) \chi(12) \chi\left(1^{\prime}\right) \chi\left(22^{\prime}\right) \pm \chi\left(1^{\prime} 1\right) \chi(12) \chi\left(2^{\prime} 1^{\prime}\right) \chi\left(22^{\prime}\right)+\chi\left(1^{\prime} 1\right) \chi\left(2^{\prime} 2\right) \chi\left(11^{\prime}\right) \chi\left(22^{\prime}\right) \\
& \pm\left(1^{\prime} 1\right) \chi\left(2^{\prime} 2\right) \chi\left(21^{\prime}\right) \chi\left(12^{\prime}\right) \pm \chi\left(2^{\prime} 1 \chi\left(1^{\prime} 2\right) \chi\left(11^{\prime}\right) \chi\left(22^{\prime}\right)+\chi\left(2^{\prime} 1\right) \chi\left(1^{\prime} 2\right) \chi\left(21^{\prime}\right) \chi\left(12^{\prime}\right)\right\} .
\end{aligned}
$$

The $\chi$ 's in these equations are defined by Eqs. (2.19) and (2.26). The notation is such that $\chi\left(1^{\prime}\right), \cdots$ is a function of $\left(\mathbf{y}_{1}{ }^{\prime}, t_{1}{ }^{\prime}\right)$, etc., and $\chi\left(1^{\prime}, 2\right), \cdots$ is a function of $\left(\mathbf{y}_{1}, t_{1}{ }^{\prime}, \mathbf{y}_{2}, t_{2}\right)$, etc.

Final evaluation of the $\Gamma$ 's is effected by performing the integrations implied by Eq. (4.5). Since these are cumbersome to do exactly, we shall restrict ourselves here to a description of certain limiting cases.

\section{THE CASE OF A MACROSCOPIC SOURCE AND DETECTORS}

We consider first the case of a "large" source. In this case the average over source points, leading to the quantity $Q$, Eq. (2.26a), must be taken into account. On evaluation of the $\Gamma$ 's [Eqs. (4.15) to (4.18)] it turns out that with each two-point $\chi$ [as in Eq. (2.26a)] there is associated a factor $\left[I_{S}\right]^{1 / 2}$, while there is no such factor for the one-point $\chi$ 's [Eq. (2.19)]. Since $I_{S}$ is a very small quantity for a macroscopic source and detectors, we may to a good approximation keep only terms not involving the two-point $\chi$ 's. There are three such terms: the first term in each of $\Gamma_{2}, \Gamma_{3}(1)$, and $\Gamma_{3}(2)$.

On evaluating these terms we obtain

$$
\begin{aligned}
\left\langle G_{\mathrm{av}}{ }^{2}\right\rangle-\left[\left\langle G_{\mathrm{av}}\right\rangle\right]^{2} \cong T\left\langle G_{1}\right\rangle_{0}\left\langle G_{2}\right\rangle_{0} M & \\
+\left\langle G_{1}\right\rangle_{0}\left\langle G_{2}\right\rangle_{0}\left[T\left\langle G_{1}\right\rangle_{0}\right. & \left.+T\left\langle G_{2}\right\rangle_{0}\right] \\
& \times\left[B_{1}(0) B_{2}(0)\right]^{2} .
\end{aligned}
$$

Here $\left\langle G_{1}\right\rangle_{0}$ is defined by Eq. (2.17) and $\left\langle G_{2}\right\rangle_{0}$ by an

\footnotetext{
${ }^{14}$ We recall that the $\Phi_{j}$ are considered to be an orthornomal set when evaluating $\langle(\Psi(0), \cdots \Psi(0))\rangle$.
}

analogous equation, and $M$ is given by

$$
\begin{aligned}
M & =\frac{V^{2}}{w_{1}^{2} w_{2}^{2} \Sigma_{1} \Sigma_{2}} \int_{1} d^{3} y_{1} \int_{1} d^{3} y_{1}{ }^{\prime} d_{2} d^{3} y_{2} \int_{2} d^{3} y_{2}{ }^{\prime} \\
& \times \int d \tau_{1} \int d \tau_{2} \frac{d \Omega}{2 \pi}\left|B_{1}(\Omega)\right|^{2}\left|B_{2}(\Omega)\right|^{2} \\
& \times e^{i \Omega\left(r_{2}-r_{1}\right)} \delta\left(\mathbf{y}_{1}^{\prime}-\mathbf{y}_{1}+V \tau_{1} \hat{y}_{1}{ }^{\prime}\right) \delta\left(\mathbf{y}_{2}{ }^{\prime}-\mathbf{y}_{2}+V \tau_{2} \hat{y}_{2}{ }^{\prime}\right) .
\end{aligned}
$$

When both counters have a uniform thickness $w_{1} \cong w_{2}=w$, this becomes

$$
M=\int \frac{d \Omega}{2 \pi}\left|B_{1}(\Omega)\right|^{2}\left|B_{2}(\Omega)\right|^{2}\left[\frac{\sin (\Omega w / 2 V)}{\Omega w / 2 V}\right]^{4} .
$$

We recall that $w$ is to be interpreted as a mean stopping distance if the particles are stopped in the detectors unless the alignment errors are larger than this. In this case, we interpret $w$ as being the alignment error, since $w / V$ appears in our equations as a measure of the spread in arrival times for particles starting at the same time from the same point.

When

$$
w / V \ll \Delta \tau_{r},
$$

[see Eq. (2.13)] we anticipate that

$$
M \approx 1 / \Delta \tau_{r} .
$$

In the other extreme limit when

$$
w / V \gg \Delta \tau_{r},
$$

the good counters are wasted and we may expect

$$
M \approx V / w .
$$


When dc blocking filters are placed in the outputs of the detectors, so $B_{1}(0)=B_{2}(0)=0$ [see Eq. (2.16a)], Eq. (5.1) becomes

$$
\left\langle G_{\mathrm{av}}{ }^{2}\right\rangle-\left[\left\langle G_{\mathrm{av}}\right\rangle\right]^{2}=T\left\langle G_{1}\right\rangle_{0}\left\langle G_{2}\right\rangle_{0} M .
$$

The "signal-to-noise ratio" in this case is

$$
\begin{aligned}
S / N \equiv\left|\left\langle G_{\text {av }}\right\rangle\right| /\left(\left\langle G_{\text {av }}{ }^{2}\right\rangle-\left[\left\langle G_{\text {av }}\right\rangle\right]^{2}\right)^{1 / 2} & =\left[T / M\left\langle G_{1}\right\rangle_{0}\left\langle G_{2}\right\rangle_{0}\right]^{1 / 2}\left|\left\langle\Delta G_{12}\right\rangle\right| I_{S},
\end{aligned}
$$

where we have used Eqs. (4.2) and (2.30). We note in passing that in the case where $M \approx\left(\Delta \tau_{r}\right)^{-1}$, Eq. (5.6) may be given a simple interpretation: We have commented that the number of coincidences $N_{c}$ is related to $\left\langle G_{12}\right\rangle$ by $\left\langle N_{c}\right\rangle=\Delta \tau_{r} T\left\langle G_{12}\right\rangle=\Delta \tau_{r}\left\langle G_{\text {av }}\right\rangle$ [Eq. (2.32)]; the quantity $\Delta \tau_{r} T\left\langle G_{1}\right\rangle\left\langle G_{2}\right\rangle$ has been called "the number of random coincidences." Thus multiplying Eq. (5.6) by $\left(\Delta \tau_{r}\right)^{2}$ and using the above definitions, we find, roughly,

$$
\left\langle N_{c}^{2}\right\rangle-\left\langle N_{c}\right\rangle^{2}=\left\langle N_{c}\right\rangle_{\text {random }} \approx\left\langle N_{c}\right\rangle \text {. }
$$

To illustrate Eq. (5.7) we set [see Eqs. (2.20) and (2.20b)]

and write

$$
\left\langle G_{1}\right\rangle_{0} \approx\left\langle G_{2}\right\rangle_{0} \approx \Sigma_{D}\left(R_{B} / 4 \pi Y^{2}\right) \eta,
$$

$\left\langle\Delta G_{12}\right\rangle_{p} \approx\left\langle G_{1}\right\rangle_{0}\left\langle G_{2}\right\rangle_{0}, \quad$ for $\Delta \tau_{r} \Delta \omega_{B} \approx 1$, (5.10a) $\left\langle\Delta G_{12}\right\rangle_{p} \approx 2 \pi\left\langle G_{1}\right\rangle_{0}\left\langle G_{2}\right\rangle_{0} / \Delta \tau_{r} \Delta \omega_{B}$, for $\left.\Delta \tau_{r} \Delta \omega_{B}\right\rangle 1$.

[These estimates may be deduced from Eq. (2.29) by considering the appropriate limits.] Then for a large source we use Eq. (2.31b) and assume Eq. (5.4) to obtain [here $\eta$ is the counter efficiency as introduced in Eq. $(2.20 \mathrm{~b})]$

$$
\begin{aligned}
& \frac{S}{N}=\left(\frac{S}{N}\right)_{a} \equiv g \frac{\lambda^{2} R_{B}}{4 \pi \Sigma_{S}}\left(T \Delta \tau_{r}\right)^{1 / 2} \eta \\
& \frac{S}{N}=\left(\frac{S}{N}\right)_{b} \equiv\left[\Delta \tau_{r} \Delta \omega_{B}\right]^{-1}\left(\frac{S}{N}\right)_{a},
\end{aligned}
$$

for cases (5.10a) and (5.10b), respectively. For photons for example, emitted by a black-body source at a temperature $\theta$ through a filter passing a narrow frequency interval $\delta \nu$ at a frequency $\nu_{0}$, we have

$$
\left(\frac{S}{N}\right)_{a}=\eta \frac{\delta \nu\left(T \Delta \tau_{r}\right)^{1 / 2}}{\exp \left(h \nu_{0} / \theta\right)-1} .
$$

\section{THE CASE OF A POINT SOURCE}

When [see Eq. (2.31)]

$$
\Sigma_{s} \Sigma_{D} \ll Y^{2} \lambda^{2},
$$

we may set $\chi(12)=\chi_{p}(12)$ in Eq. (2.26a) and in Eqs. (4.15) to (4.18). In this case a general evaluation is tortuous and we distinguish several limiting cases. [In the spirit of an approximate description we now set the spin weighting factor $g$ of Eq. (2.29) equal to unity.] Case I [see Eqs. (2.6) and (2.13)]:

$$
V \ll w \Delta \omega_{B}, \quad V \Delta \tau_{r} \ll w,
$$

the case of "broad-beam spectrum" and "fast electronics." In this case we may take $B_{1}(0)=B_{2}(0)=1$, as in Eq. (2.16a), without serious loss of counting accuracy. Then, we obtain from Eqs. (4.7) and (4.15) to (4.18)

$$
\begin{aligned}
& \left\langle G_{\mathrm{av}}{ }^{2}\right\rangle-\left[\left\langle G_{\mathrm{av}}\right\rangle\right]^{2} \cong\left\langle G_{1}\right\rangle\left\langle G_{2}\right\rangle(T V / w)+\left\langle G_{1}\right\rangle\left\langle G_{2}\right\rangle \\
& \quad \times\left\{T\left\langle G_{1}\right\rangle\left[1 \pm 2 \frac{\left\langle G_{2}\right\rangle}{\Delta \omega_{B}}\right]+T\left\langle G_{2}\right\rangle\left[1 \pm 2 \frac{\left\langle G_{1}\right\rangle}{\Delta \omega_{B}}\right]\right\} .
\end{aligned}
$$

Case II:

$$
V \ll w \Delta \omega_{B}, \quad V \Delta \tau_{r} \gg w,
$$

the case of a broad beam spectrum and slow electronics. In this case it is convenient to replace several functions having similar properties by a qualitatively defined quantity $\epsilon_{r}(\tau)$, having no more precise definition than

$$
\begin{aligned}
\epsilon_{r}(\tau)=1 & \text { for } & \left|V \tau-\left(Y_{2}-Y_{1}\right)\right| \ll V \Delta \tau_{r} \\
=0 & \text { for } & \left|V \tau-\left(Y_{2}-Y_{1}\right)\right| \gg V \Delta \tau_{r} .
\end{aligned}
$$

For case II we obtain

$$
\begin{aligned}
& {\left[\left\langle G_{\mathrm{av}}{ }^{2}\right\rangle-\left[\left\langle G_{\mathrm{av}}\right\rangle\right]^{2}\right] \cong\left\langle G_{1}\right\rangle_{0}\left\langle G_{2}\right\rangle_{0}\left(T / \Delta \tau_{r}\right)} \\
& +\left\langle G_{1}\right\rangle_{0}\left\langle G_{2}\right\rangle_{0}\left[T\left\langle G_{1}\right\rangle_{0}+T\left\langle G_{2}\right\rangle_{0}\right] \\
& \times\left\{B_{1}{ }^{2}(0) B_{2}{ }^{2}(0) \pm 2 \frac{B_{1}(0) B_{2}(0)}{\Delta \tau_{r} \Delta \omega_{B}} \epsilon_{r}(\tau) \pm \frac{1}{\Delta \tau_{r} \Delta \omega_{B}}\right\} \\
& +\left[\left\langle G_{1}\right\rangle_{0}\left\langle G_{2}\right\rangle_{0}\right]^{2}\left[\frac{T}{\Delta \omega_{B}}\right]\left\{ \pm 4 B_{1}{ }^{2}(0) B_{2}{ }^{2}(0)\right. \\
& \left.+8 \frac{B_{1}(0) B_{2}(0)}{\Delta \tau_{r} \Delta \omega_{B}} \epsilon_{r}(\tau)+\frac{1+\epsilon_{r}(\tau)}{\Delta \tau_{r} \Delta \omega_{B}}\right\}
\end{aligned}
$$

Case III:

$$
V \gg w \Delta \omega_{B}, \quad V \Delta \tau_{r} \ll w, \quad \Delta \tau_{r} \Delta \omega_{B} \ll 1,
$$

the case of a narrow beam spectrum and fast electronics. In this case we again take $B_{1}(0)=B_{2}(0)=1$ and introduce the "function" $\epsilon_{B}(\tau)$ [analogous to the expression (6.4)]:

$$
\begin{aligned}
\epsilon_{B}(\tau)=1 \text { for } \quad\left|V \tau-\left(Y_{2}-Y_{1}\right)\right| \ll V / \Delta \omega_{B}, \\
=0 \text { for } \quad\left|V \tau-\left(Y_{2}-Y_{1}\right)\right| \gg B / \Delta \omega_{B} .
\end{aligned}
$$

Then for case III,

$$
\begin{aligned}
& \left\langle G_{\mathrm{av}}{ }^{2}\right\rangle-\left[\left\langle G_{\mathrm{av}}\right\rangle\right]^{2} \cong\left\langle G_{1}\right\rangle\left\langle G_{2}\right\rangle(V T / w)\left[1 \pm \epsilon_{B}(\tau)\right] \\
& \quad+\left\langle G_{1}\right\rangle\left\langle G_{2}\right\rangle\left[T\left\langle G_{1}\right\rangle+T\left\langle G_{2}\right\rangle\right](1 \pm 1)\left[1+2 \epsilon_{B}(\tau)\right] \\
& \quad+\left[\left\langle G_{1}\right\rangle\left\langle G_{2}\right\rangle\right]^{2}\left[1 \pm 4+(9 \pm 6) \epsilon_{B}(\tau)\right]\left(T / \Delta \omega_{B}\right) .
\end{aligned}
$$




\section{SCATTERING EXPERIMENTS}

We now consider a scattering experiment, as illustrated in Fig. 5. The scattered-beam particles are counted in two detectors whose outputs are mixed, with a relative time delay $\tau$, in a correlator. It was shown in I that the one-point $\chi$ [Eq. (2.19)] in this case has the form ${ }^{15}$

$$
\chi(1)=(2 \pi)^{3} \frac{N_{B}}{\bar{n}} \int d \omega g(\omega)\left\langle\left|\phi_{j}\left(q, \mathbf{y}_{1}, t_{1}\right)\right|^{2}\right\rangle_{t, S} .
$$

Here " $\langle\cdots\rangle_{t}$ " denotes an average over the target wave functions $g_{0}$ and then a statistical ensemble average over target states. The additional subscript " $S$ " on " $\langle\cdots\rangle$ " represents an additional average over the source volume:

$$
\langle\cdots\rangle_{S}=\int_{S} \frac{d^{3} s}{v_{S}} \cdots,
$$

as in Eq. (2.26c). The quantity $\phi_{j}(q, \mathbf{y}, t)$ is the wave function for a scattered particle. It is, at large distances from the target,

$$
\begin{aligned}
\phi_{j}(q, \mathbf{y}, t)=\left((2 \pi)^{-3 / 2} / y\right) & \\
& \quad \times \exp \left\{i\left[q\left(y+d_{j}\right)-\omega t\right]\right\} F\left(\hat{y}, \hat{d}_{j}\right),
\end{aligned}
$$

where $\mathcal{F}$ is the scattering amplitude and $\mathbf{d}_{j}$ is a vector from a reference point in the target to the source point of particle $j$ in the source. ${ }^{16}$

The two-point $\chi[\mathrm{Eq} .(2.26)]$ now has the form, derived in I,

$$
\begin{aligned}
\chi(12)=(2 \pi)^{3} \frac{N_{B}}{\bar{n}} \int d \omega & g(\omega) \\
& \times\left\langle\phi_{j}{ }^{*}\left(q, \mathbf{y}_{1}, t_{1}\right) \phi_{j}\left(q, \mathbf{y}_{2}, t_{2}\right)\right\rangle_{t, S} .
\end{aligned}
$$

Making use of (7.3), we obtain

$$
\begin{aligned}
\chi(1)=\frac{N_{B}}{\bar{n} y_{1}{ }^{2}} \int d \omega & g(\omega) \\
& \quad \times\langle| F\left(q, \hat{y}_{1},\left.\hat{d}\right|^{2}\right\rangle_{t, S} \equiv\left(N_{B} / \bar{n} y_{1}{ }^{2}\right) \tilde{\sigma}_{1},
\end{aligned}
$$

and

$$
\begin{aligned}
\chi(12)=\frac{N_{B}}{\bar{n} y_{1} y_{2}} \int d \omega g(\omega) & \exp \left\{i\left[q\left(y_{2}-y_{1}\right)-\omega\left(t_{2}-t_{1}\right)\right]\right\} \\
& \times\left\langle F^{*}\left(q, \mathbf{y}_{1}, \hat{d}\right) \mathcal{F}\left(q, \mathbf{y}_{2}, \hat{d}\right)\right\rangle_{t, S}, \quad
\end{aligned}
$$

where we have defined the average cross section $\bar{\sigma}_{1}$ in the second writing of Eq. (7.5).

From Eq. (2.18) we obtain the mean counting rate for a single detector as

$$
\begin{aligned}
\left\langle G_{1}\right\rangle & =B_{1}(0)\left(\left(\Sigma_{1} w_{1} \gamma_{1}\right) / Y_{1}^{2}\right) N_{B} \bar{\sigma}_{1} \\
& =B_{1}(0) \Sigma_{1}\left(\bar{\sigma}_{1} / Y_{1}^{2}\right)\left(V N_{B}\right),
\end{aligned}
$$

\footnotetext{
${ }^{15}$ We now depart slightly from the notation used in $\mathrm{I}$. The quantity called $\chi$ here was written as $\left(\left(g_{0}, \chi g_{0}\right)\right)$ in I.

${ }_{16}$ The scattering amplitude $\mathcal{F}$ was evaluated at the retarded time $t-(y / V)$ in I. We shall not include this correction here.
}

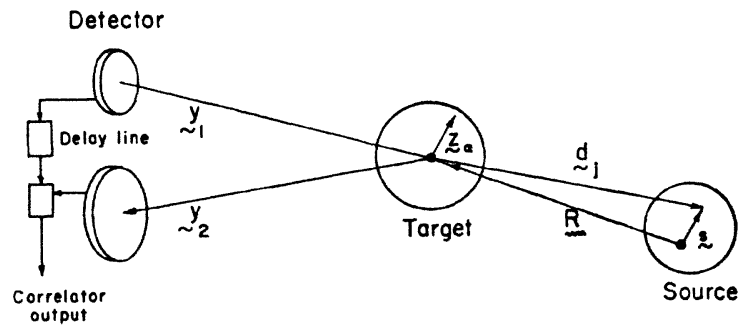

FIG. 5. Intensity correlations for a scattering experiment.

using Eq. (2.11). We see that

$$
F_{T}=V N_{B}
$$

is to be interpreted as the beam flux incident on the target. [To account for detector efficiency, a factor $\eta$, Eq. (2.20b), should be included on the right-hand side of Eq. (7.7).]

From Eq. (2.24) and (2.27) we see that

$$
\begin{aligned}
& \left\langle\Delta G_{12}\right\rangle= \pm \frac{\left\langle G_{1}\right\rangle_{0}\left\langle G_{2}\right\rangle_{0}}{\bar{\sigma}_{1} \bar{\sigma}_{2}} \int_{1} \frac{d^{3} y_{1}}{\Sigma_{1} w_{1}} \int \frac{d^{3} y_{2}}{\Sigma_{2} w_{2}} \\
& \quad \times \int d \omega d \omega^{\prime} g(\omega) g\left(\omega^{\prime}\right) B_{1}\left(\omega^{\prime}-\omega\right) B_{2}\left(\omega-\omega^{\prime}\right) \\
& \quad \times \exp \left\{i\left[\left(q-q^{\prime}\right)\left(y_{2}-y_{1}\right)-\left(\omega-\omega^{\prime}\right)\left(T_{2}-T_{1}\right)\right]\right\} \\
& \quad \times\left\langle F^{*}\left(q, \hat{y}_{1}, \hat{d}\right) \mathfrak{F}\left(q, \hat{y}_{2}, \hat{d}\right)\right\rangle_{t, S}\left\langle\mathcal{F}\left(q^{\prime}, \hat{y}_{1}, \hat{d}^{\prime}\right) \mathfrak{F}^{*}\left(q^{\prime}, \hat{y}_{2}, \hat{d}^{\prime}\right)\right\rangle_{t, S}
\end{aligned}
$$

where a different source point average [Eq. (7.2)] is implied for the vectors $\mathbf{d}$ and $\mathbf{d}^{\prime}$.

Let us consider now a specific case to illustrate Eq. (7.9). We suppose the target to be homogeneous and composed of a large number $N$ of identical scatterers. We shall write $f$ for the scattering amplitude of any one of these and, as in I, write

$$
F(q, \hat{y}, \hat{d})=f \sum_{\alpha=1}^{N} \exp \left[-i q(\hat{y}+\hat{d}) \cdot \mathbf{Z}_{\alpha}\right]
$$

where $\boldsymbol{Z}_{\alpha}$ is the coordinate of a given scatterer. We shall suppose that, as is the case for a gas or liquid, the scatters are uncorrelated when separated by a distance large compared to $\Omega_{c}$, the "range of correlation" in the target. We shall also suppose that we may set $q=p$ in $F$.

When $R_{c}$ satisfies the conditions that

$$
p \Sigma_{S^{1 / 2}} \mathcal{R}_{c} \ll R, \quad p \Sigma_{d}{ }^{1 / 2} \mathcal{R}_{c} \ll Y,
$$

where $R$ is the distance from target to source, we may neglect the finite size of the source and write

$$
\chi(12)=\chi_{p}(12)\left\langle\mathcal{F}^{*}(1) \mathcal{F}(2)\right\rangle_{t} Q_{T}(12) .
$$

Here $\chi_{p}(12)$ is defined by Eq. $(2.26 \mathrm{~b})$ and [see I for 
further discussion of the notation]

$$
\begin{aligned}
&\left\langle F^{*}(1) \mathcal{F}(2)\right\rangle_{t}=f^{*}\left(\hat{y}_{1}\right) f\left(\hat{y}_{2}\right) \\
& \times \int d^{3} \hat{d} d^{3} r\left\langle n\left(\mathbf{x}+\frac{1}{2} \mathbf{r}, t_{1}\right) n\left(\mathbf{x}-\frac{1}{2} \mathbf{r}, t_{2}\right)\right\rangle \\
& \quad \times \exp \left\{i p\left[\frac{1}{2}\left(\hat{y}_{1}+\hat{y}_{2}\right)-\hat{R}\right] \cdot \mathbf{r}\right\},
\end{aligned}
$$

where $\mathbf{R}$ is a vector from the fixed reference point in the target to a fixed point in the source and $\left\langle n\left(\mathbf{x}^{\prime}, t_{1}\right) n\left(\mathbf{x}, t_{2}\right)\right\rangle$ is the Van Hove correlation function for the target. The quantity $Q_{T}(12)$ in Eq. (7.12) is

$$
Q_{T}(12)=\int \frac{d^{3} Z}{v_{T}} \exp \left[i p\left(\hat{y}_{1}-\hat{y}_{2}\right) \cdot \mathbf{Z}\right],
$$

integrated over the volume of the target.

The quantity (7.9) now becomes

$$
\left\langle\Delta G_{12}\right\rangle=\left\langle\Delta G_{12}\right\rangle_{p} I_{S},
$$

where $I_{S}$ is defined by Eqs. (2.31), but with $Q(12)$ replaced by $Q_{T}(12)$ and thus $\Sigma_{S}$ replaced by the target area $\Sigma_{T}$ in $(2.31 \mathrm{~b})$, and

$$
\begin{aligned}
& \left\langle\Delta G_{12}\right\rangle_{p}= \pm \frac{\left\langle G_{1}\right\rangle_{0}\left\langle G_{2}\right\rangle_{0}}{\bar{\sigma}_{1} \bar{\sigma}_{2}} \int_{1} \frac{d^{3} y_{1}}{\Sigma_{1} w_{1}} \int_{2} \frac{d^{3} y_{2}}{\Sigma_{2} w_{2}} \int d \omega d \omega^{\prime} \\
& \times g(\omega) g\left(\omega^{\prime}\right) B_{1}\left(\omega^{\prime}-\omega\right) B_{2}\left(\omega-\omega^{\prime}\right) \exp \left\{i \left[\left(q-q^{\prime}\right)\left(y_{2}-y_{1}\right)\right.\right. \\
& \left.\left.\quad-\left(\omega-\omega^{\prime}\right)\left(T_{2}-T_{1}\right)\right]\right\}\left|\left\langle\mathcal{F}^{*}(1) \mathscr{F}(2)\right\rangle_{t}\right|^{2} . \quad(7.16)
\end{aligned}
$$

Fluctuations in

$$
\left\langle G_{\mathrm{av}}\right\rangle=T\left[\left\langle G_{1}\right\rangle\left\langle G_{2}\right\rangle+\left\langle\Delta G_{12}\right\rangle\right]
$$

may be evaluated from the general analysis of Sec. IV. When the target is large enough that $I_{T} \ll 1$, we may use Eq. (5.1) to evaluate these. The detector efficiency may be taken into account by including in $\left\langle G_{1}\right\rangle_{0}$ and $\left\langle G_{2}\right\rangle_{0}$ an efficiency factor (or factors) $\eta$, as in Eq. (2.20b).

\title{
Position Operators in Relativistic Single-Particle Theories*
}

\author{
A. Sankaranarayanan and R. H. Good, JR. \\ Institute for Atomic Research and Department of Physics, Iowa State University, Ames, Iowa
}

(Received 21 May 1965)

\begin{abstract}
This paper gives formulas for position operators that apply uniformly for all spins of particle. The threevector $\mathbf{X}$, of the Newton-Wigner and Foldy type, is treated first. Then it is shown that, although $\mathbf{X}$ has complicated Lorentz transformation properties, it is linearly related to a certain four-vector $Y_{\mu}$ which is built up from the Poincaré group generators. The four-vector is the generalization of the classical notion of the component of the position four-vector in the direction perpendicular to the world line of the particle.
\end{abstract}

\section{INTRODUCTION}

$\mathbf{R}$ ECENTLY a formulation of the theory of a free particle with mass and arbitrary spin was given ${ }^{1}$ in which there is such a complete parallel with Dirac's theory for an electron-positron that all the known discussions for a spin $-\frac{1}{2}$ particle can be extended to particles with higher spins. The purpose of the present paper is to make this extension for the study of position and to develop formulas for three-vector and four-vector position operators that apply uniformly for all spins.

The special features of the description of free particles developed in Ref. 1 are that there are no auxiliary conditions on the wave function and that the wave-function components are spinors, so that the value of the wave function at a point in space-time in one Lorentz frame determines the value in all Lorentz frames. It is closely

\footnotetext{
* This research was done in the Ames Laboratory of the U.S. Atomic Energy Commission.

${ }_{1}$ D. L. Weaver, C. L. Hammer, and R. H. Good, Jr., Phys. Rev. 135, B241 (1964).
}

related to Weinberg's ${ }^{2}$ formulation and Foldy's. ${ }^{3}$ In fact there is an operator, which is a generalization of the notion of the rest-to-lab Lorentz transformation, which carries Foldy's wave function into the wave function of Ref. 1. Consequently properties of operators in Foldy's theory can be similarity transformed into the present formulation.

For many of the observable quantities, such as momentum, energy, and angular momentum, the corresponding operators are simply the inhomogeneous Lorentz group generators. The situation is not so straightforward since position and other considerations have to be made. Desirable properties for a position operator $\mathbf{X}$ are that (i) it should be Hermitian with respect to the appropriate Lorentz-invariant inner product for each spin; (ii) it should fulfil the commutation rules

$$
\begin{aligned}
{\left[X_{i}, X_{j}\right] } & =0, \\
{\left[X_{i}, p_{j}\right] } & =i \delta_{i j},
\end{aligned}
$$

2. Weinberg, Phys. Rev. 133, B1318 (1964).

${ }^{3}$ L. L. Foldy, Phys. Rev. 102, 568 (1956). 